\title{
THE SEMICENTRE OF A GROUP ALGEBRA
}

\author{
by PAUL WAUTERS
}

(Received 12th February 1997)

\begin{abstract}
We study the semicentre of a group algebra $K[G]$ where $K$ is a field of characteristic zero and $G$ is a polycyclic-by-finite group such that $\Delta(G)$ is torsion-free abelian. Several properties about the structure of this ring are proved, in particular as to when is the semicentre a UFD. Examples are constructed when this is not the case. We also prove necessary and sufficient conditions for every normal element of $K[G]$ which belongs to $K[\Delta(G)]$ to be the product of a unit and a semi-invariant.
\end{abstract}

1991 Mathematics subject classification: 16S34, 16U70, $16 \mathrm{P} 40$.

\section{Introduction}

Let $K$ be a field of characteristic zero and $G$ a polycyclic-by-finite group such that $K[G]$ is a prime Noetherian ring. This type of group algebra is often compared with a universal enveloping algebra $U(L)$ of a finite dimensional Lie algebra $L$ over a field of characteristic zero. A number of basic properties are similar, but we show that there are also some striking differences between the semicentre of both types of rings. To be a little more concrete, in both cases the semicentre is a commutative domain graded by an abelian monoid. In the case of $U(L)$ it is well-known and obvious that this monoid is torsion-free abelian while for $K[G]$ we prove this is a finite group. We show that this difference in the grading monoid implies that the semicentre of the classical ring of quotients of $K[G]$ behaves quite differently from the case of $U(L)$ (cf. [7]). The main difference is perhaps the property of being a UFD: the semicentre of $U(L)$ is always a UFD (cf. $[9,13]$ ) and we observe when it is a UFD in case of a group algebra. This is an immediate consequence of a result of $\mathbf{M}$. Lorenz on rings of multiplicative invariants [10]. In computing the semicentre of $K[G]$ in a number of examples we show e.g., that the property of being a UFD of the semicentre does not depend only on $G$ but also on the field $K$.

Throughout this paper $K$ is a field of characteristic zero, $K^{*}$ denotes $K \backslash\{0\}$ and $\bar{K}$ is the algebraic closure of $K$. The group of elements of a group $G$ which have only finitely many conjugates is denoted by $\Delta(G)$ or shortly by $\Delta$. Then $K[G]$ is a prime ring if and only if $\Delta(G)$ is torsion-free abelian, by Connell's Theorem (see e.g., [19, 4.2.10]). Unless explicitly mentioned, all groups are polycyclic-by-finite such that $\Delta(G)$ is torsion-free abelian. 
Acknowledgements. The author is grateful to D. S. Passman who read the first version of this paper and gave several helpful comments and ideas. The author also thanks M. Lorenz for sending a preprint of [10].

\section{Definitions and basic properties}

In [22] $M$. Smith makes a distinction between semi-invariant ring and semicentre. To improve readability, we combine the definitions as mentioned in [22, p. 1283 and p. 1290].

Definition 1.1. Let $R$ be a $K$-algebra and $G$ a subgroup of units of $R$. Then $G$ acts on $R$ by inner automorphisms. If $r \in R$ and $g \in G$, denote $g r g^{-1}$ by $r^{g}$.

(1) Let $0 \neq r \in R$ and $\lambda \in \operatorname{Hom}\left(G, K^{*}\right)$. Then $\alpha$ is said to be a semi-invariant with weight $\lambda$ if for each $g \in G r^{g}=\lambda(g) r$.

(2) If $\lambda \in \operatorname{Hom}\left(G, K^{*}\right)$ we denote the set of semi-invariants with weight $\lambda$ together with 0 by $R_{\lambda}$. If $R_{\lambda} \neq 0, \lambda$ is a weight. The set of all weights is denoted by $\Lambda(G, K)$ (or shortly by $\Lambda(G)$ ).

(3) The semicentre of $R$, denoted by $\mathrm{Sz} R$, is defined as

$$
\mathrm{SzR}=\sum_{\lambda \in \Lambda(G)} R_{l}
$$

Of course, if $R=K[G]$ is a group algebra, $G$ acts on $K[G]$ by inner automorphisms and the foregoing definition makes sense.

Proposition 1.2. (1) $\mathrm{SzK}[G]=\oplus_{\lambda \in \Lambda(G)}(K[G])_{\lambda}$ is a subring of $K[\Delta(G)]$;

(2) $\mathrm{SzK}[G]$ is a commutative domain;

(3) $\Lambda(G)$ is an abelian cancellative monoid and $\mathrm{SzK}[G]$ is a $\Lambda(G)$-graded ring;

(4) A semi-invariant is a normal element.

Proof. (1) As mentioned in [22], a standard linear algebra argument shows that $\sum_{\lambda \in \Lambda(G)}(K[G])_{\lambda}$ is in fact a direct sum $\oplus_{\lambda \in \Lambda(G)}(K[G])_{\lambda}$. If $\alpha$ is a semi-invariant with weight $\lambda$, then $g \alpha g^{-1}=\lambda(g) \alpha$ for all $g \in G$, hence supp $\alpha \subset \Delta(G)$. Therefore $\operatorname{SzK}[G] \subset K[\Delta(G)]$.

(2) and (3) follow directly from (1) because $K[\Delta]$ is a commutative domain.

(4) If $\alpha$ is a semi-invariant with weight $\lambda$, then for all $g \in G g \alpha=\alpha(\lambda(g) g)$ and $\alpha g=\left(\lambda(g)^{-1} g\right) \alpha$. So $K[G] \alpha=\alpha K[G]$, i.e., $\alpha$ is a normal element.

Lemma 1.3. If $\lambda \in \Lambda(G)$, then $\lambda\left(G^{\prime}\right)=\lambda\left(C_{G}(\Delta)\right)=1$. 
Proof. (1) If $g, h \in G$, then $\lambda([g, h])=\lambda\left(g h g^{-1} h^{-1}\right)=\lambda(g) \lambda(h) \lambda(g)^{-1} \lambda(h)^{-1}=1$.

(2) Let $\alpha$ be a semi-invariant with weight $\lambda$. If $g \in C_{G}(\Delta)$, i.e., $g h=h g$ for all $h \in \Delta$, then $\alpha^{g}=g \alpha g^{-1}=\alpha$ since $\alpha \in K[\Delta]$. On the other hand $\alpha^{g}=\lambda(g) \alpha$. Thus $\lambda(g)=1$.

Lemma 1.4. (1) $G / C_{G}(\Delta)$ is a finite group;

(2) $C_{G}(\Delta)=C_{G}(x)$ for some $x \in \Delta$.

Proof. (1) Clearly $C_{G}(\Delta)$ is a normal subgroup of $G$. On the other hand, $\Delta$ is a subgroup of $G$, thus finitely generated, say by $x_{1}, \ldots, x_{k}$. Then $C_{G}(\Delta)=\bigcap_{i=1}^{k} C_{G}\left(x_{i}\right)$. Since $x_{i} \in \Delta,\left(G: C_{G}\left(x_{i}\right)\right)$ is finite and hence $\left(G: C_{G}(\Delta)\right)$ is finite by e.g., [19, Lemma 4.1.3].

(2) This is proved in [16, Lemma 2].

We now construct all semi-invariants having a certain weight $\lambda$. This is partially based on [15, Lemma 3]. First note that if $\alpha=\sum a_{g} g \in(K[G])$, and $x \in \operatorname{supp} \alpha$ with (finite) conjugacy class $C_{x}$, then obviously $\alpha_{(x)}=\sum_{g \in C_{x}} a_{g} g$ is a semi-invariant having weight $\lambda$ and $\alpha$ is a sum of such $\alpha_{(x)}$. Therefore it suffices to construct semi-invariants $\alpha$ such that $\operatorname{supp} \alpha$ is precisely a conjugacy class.

Lemma 1.5. Let $\lambda \in \operatorname{Hom}\left(G, K^{*}\right)$.

(1) If $C_{G}(x) \subset \operatorname{ker} \lambda$ for some $x \in \Delta(G)$ and $T$ denotes a left transversal for $C_{G}(x)$ in $G$, then $\alpha=\sum_{t \in T} \lambda(t)^{-1} x^{t}$ is a semi-invariant with weight $\lambda$ (note that $\operatorname{supp} \alpha=C_{x}$ ).

(2) Conversely, if $\alpha \in(K[G])$, such that supp $\alpha$ equals precisely a conjugacy class of an element $x$, then $\alpha=a\left(\sum_{t \in T} \lambda(t)^{-1} x^{t}\right)$ where $a \in K^{*}$ and $T$ is a left transversal for $C_{G}(x)$ in $G$.

Proof. (1) This is proved in [15, Lemma 3], up to a slight difference in notation.

(2) Conversely, let $\alpha \in(K[G])_{\lambda}$, such that supp $\alpha=C_{x}$ for some $x$. Let $T$ be a left transversal for $C_{G}(x)$ in $G$. Write $T=\left\{t_{1}=1, t_{2}, \ldots, t_{n}\right\}$ and $\alpha=\sum_{i=1}^{n} a_{i} x^{t_{i}}$. If $j \neq 1$, then

$$
\alpha^{t_{j}}=\sum_{i=1}^{n} a_{i} x^{t_{1} t_{i}} \text { and } \alpha^{t_{j}}=\lambda\left(t_{j}\right) \alpha=\sum_{i=1}^{n} a_{i} \lambda\left(t_{j}\right) x^{t_{i}}
$$

In particular, $a_{1}=a_{j} \lambda\left(t_{j}\right)$ or $a_{j}=\lambda\left(t_{j}^{-1}\right) a_{1}$. Therefore

$$
\alpha=a_{1}\left(\sum_{i=1}^{n} \lambda\left(t_{i}^{-1}\right) x^{t_{i}}\right)
$$

Proposition 1.6. $\Lambda(G) \cong \operatorname{Hom}\left(G / C_{G}(\Delta), K^{*}\right)$ and thus $\Lambda(G)$ is a finite abelian group. 
Proof. Since $\lambda\left(C_{G}(\Delta)\right)=1$ if $\lambda \in \Lambda(G)$ by Lemma 1.3, it is straightforward to check that the map

$$
\begin{aligned}
\Lambda(G) \rightarrow \operatorname{Hom}\left(G / C_{G}(\Delta), K^{*}\right): \lambda \mapsto \bar{\lambda}: G / C_{G}(\Delta) & \rightarrow K^{*} \\
\bar{g} & \mapsto \lambda(g)
\end{aligned}
$$

is a well-defined injective homomorphism of monoids. To prove the surjectivity, let $\lambda \in \operatorname{Hom}\left(G / C_{G}(\Delta), K^{*}\right)$, then with

$$
\underbrace{G \rightarrow G / C_{G}(\Delta) \stackrel{\lambda}{\rightarrow} K^{*}}_{\mu},
$$

$\mu \in \operatorname{Hom}\left(G, K^{*}\right)$ such that $\mu\left(C_{G}(\Delta)\right)=1$. Combining Lemma 1.4(2) and Lemma 1.5(1), there exists a semi-invariant $\alpha$ with weight $\mu$. Thus $\mu \in \Lambda(G)$ and $\bar{\mu}=\lambda$. In particular this shows that $\Lambda(G)$ is a finite abelian group.

Corollary 1.7. $\mathrm{Sz} K[G]=Z K[G]$ if and only if $\operatorname{Hom}\left(G / C_{G}(\Delta), K^{*}\right)=\{1\}$.

Of course, if $G$ is nilpotent (and such that $G$ is polycyclic-by-finite and $\Delta(G)$ is torsion-free abelian), then $C_{G}(\Delta)=G$ since $\Delta(G)=Z(G)$ in this case (cf. e.g., [19, Lemma 11.4.3]). However, since $\Delta(G)=Z(G)$, we immediately have $K[Z(G)] \subset$ $Z(K[G]) \subset \operatorname{SzK}[G] \subset K[\Delta(G)]=K[Z(G)]$ and thus $\operatorname{SzK}[G]=Z K[G]$. Example 6.4 shows however that $\operatorname{SzK} G$ can also be equal to $Z K[G]$ if $G$ is not nilpotent.

The next proposition shows that every finite abelian group can be the group of weights of some group algebra.

Proposition 1.8. Every finite abelian group is the group of weights of some group algebra $K[G]$ where $K$ is algebraically closed.

Proof (due to D. S. Passman; the original proof of the author was longer). Let $A$ be an infinite cyclic group and $H$ a finite abelian group. Let $G$ be the wreath product of $A$ by $H$, denoted $G=A \imath H$. Then $G=W \times{ }_{\sigma} H$, the semidirect product of $W$ and $H$, where $W$ is a direct product of copies of $A$ indexed by $H$. Using the fact that $A$ is abelian and infinite and that $H$ is finite abelian, it is straightforward to conclude that $\Delta(G)=W=C_{G}(W)$. Thus $G / C_{G}(\Delta) \cong H$ and using Proposition 1.6 we obtain that $\Lambda(G) \cong \operatorname{Hom}\left(H, K^{*}\right) \cong H$ because $K$ is algebraically closed.

Remark 1.9. Proposition 1.8 does not hold if $K$ is not algebraically closed. For example, let $K=\mathbb{R}$; then the cyclic group of order 4 cannot be the group of weights of some group algebra $\mathbb{R}[G]$. For if such a group exists and $\alpha$ is a semi-invariant with weight $\lambda$, then for all $g \in G \lambda\left(g^{4}\right)=\lambda(g)^{4}=1$ since $\lambda \in C_{4}$. Within $\mathbb{R}$ this means $\lambda(g)$ is either 1 or -1 . Thus $\lambda^{2}=1$ within $\Lambda(G)$. Hence $\Lambda(G)$ is not isomorphic to $C_{4}$. 


\section{The semicentre of $Q_{\mathrm{cl}}(K[G])$}

2.1. Since $K[G]$ is prime Noetherian, it has a classical ring of quotients $Q_{c l}(K[G])$ which is simple Artinian. We will denote this ring shortly by $Q$. The group $G$ is obviously contained in the units of $Q$ and so the definition of semi-invariant and semicentre of $Q$ makes sense by Definition 1.1. In this case we will denote the set of weights by $\Lambda_{Q}(G, K)$ (or shortly $\Lambda_{Q}(G)$ ).

Remarks 2.2. (1) As mentioned in proposition 1.2 and its proof, $\sum_{\lambda \in \Lambda_{Q}(G)} Q_{i}$ is in fact a direct sum $\bigoplus_{\lambda \in \Lambda_{Q}(G)} Q_{\lambda}$.

(2) Let $\lambda \in \Lambda_{Q}(G)$ and $0 \neq \alpha \in Q$. By definition of a semi-invariant $Q \alpha=\alpha Q$ and hence $\alpha$ is invertible. In particular $\Lambda_{Q}(G)$ is a monoid. From $g \alpha g^{-1}=\lambda(g) \alpha$ one obtains $g \alpha^{-1} g^{-1}=\lambda(g)^{-1} \alpha^{-1}$ for all $g \in G$, i.e., $\alpha^{-1}$ is a semi-invariant with weight $\lambda^{-1}$. Thus $\Lambda_{Q}(G)$ is an (abelian) group. Clearly $\Lambda(G) \subset \Lambda_{Q}(G)$; we will show later on that these two groups coincide.

(3) By (1) and (2) $\mathrm{Sz} Q$ is a $\Lambda_{Q}(G)$-graded ring and ( $\left.\mathrm{Sz} Q\right)_{1}=Z\left(Q_{c l}(K[G])\right)$ which equals $Q_{c l}(Z K[G])$ by $[19$, Theorem 4.4 .5$]$.

The following lemma is just a basic observation.

Lemma 2.3. Let $\alpha \in Q_{\lambda}$. If $u, v \in K[G]$, u regular, then $\left(u^{-1} v\right) \alpha=\alpha\left(\lambda^{\#}(u)^{-1} \lambda^{\#}(v)\right)$ where $\lambda^{\#}\left(\sum u_{g} g\right)=\sum u_{g} \lambda(g) g$ (cf. [15, p. 397] for the notation $\left.\lambda^{\#}\right)$.

Proposition 2.4. $\mathrm{Sz} Q$ is the localisation of $\mathrm{SzK}[G]$ at the nonzero central elements of $K[G]$, i.e., $\mathrm{Sz} Q=(\mathrm{SzK}[G])_{z K[G \backslash(0)}$.

Proof. Obviously $(\mathrm{SzK}[G])_{Z K[G \backslash 0\}}$ is contained in $\mathrm{Sz} Q$. We show the converse inclusion. Let $\alpha \in Q_{\lambda}$. Denote $I_{l}=\{u \in K[G] \mid u \alpha \in K[G]\}$ and $I_{r}=\{u \in K[G] \mid \alpha u \in K[G]\}$. By Lemma $2.3 I_{1}$ and $I_{r}$ are nonzero twosided ideals of $K[G]$. Thus $\alpha \in Q_{s}(K[G])$, the symmetric Martindale ring of quotients of $K[G]$. If $K[G]$ is prime and $G$ is polycyclicby-finite, then $Q_{s}(K[G])=K[G]_{Z K|G| \backslash(0)}$ by [21, Theorem 11.12] (or [20, Corollary 7.8] for a detailed proof). Thus $z \alpha \in K[G]$ for some nonzero central element $z$ in $K[G]$. Obviously $z \alpha \in(K[G])$, which shows the result.

Corollary 2.5. (1) $\Lambda_{Q}(G)=\Lambda(G)$; in particular, $\Lambda_{Q}(G)$ is a finite abelian group;

(2) $\mathrm{SzQ}$ is a commutative domain;

(3) $\mathrm{Sz} Q \cong F^{t}[\Lambda(G)]$, a twisted group algebra, where $F=Q_{c l}(Z K[G])$;

(4) $\mathrm{Sz} Q=Q(\mathrm{Sz} K[G])$, the field of fractions of $\mathrm{Sz} K[G]$.

Proof. (1) and (2) follow immediately from Proposition 2.4 .

(3) Let $\lambda \in \Lambda(G)$ and choose a nonzero element $\alpha_{\lambda} \in Q_{\lambda}$. Since $\alpha_{\lambda}^{-1} \in Q_{\lambda-1}$ (cf. 
Remarks 2.2 (2)) $Q_{\lambda} \alpha_{\lambda}^{-1} \subset F$ and thus $Q_{\lambda} \subset F \alpha_{\lambda}$. The converse inclusion is trivial and therefore $Q_{\lambda}=F \alpha_{\lambda}$. This shows that $S z Q=\oplus_{l \in \Lambda(G)} F \alpha_{\lambda}$ which is obviously isomorphic to $F^{t}[\Lambda(G)]$.

(4) By (2) and (3) $\mathrm{Sz} Q$ is a commutative domain and finite dimensional over $F$; a classical argument shows that $\mathrm{Sz} Q$ is a field. Moreover,

$$
\mathrm{Sz} K[G] \subset \mathrm{Sz} Q=(\mathrm{Sz} K[G])_{z K(G)(0)} \subset Q(\mathrm{Sz} K[G]) .
$$

Since $\mathrm{Sz} Q$ is a field, every nonzero element of $\mathrm{Sz} K[G]$ is invertible in $\mathrm{Sz} Q$ and thus $\mathrm{Sz} Q=Q(\mathrm{SzK} K[G])$.

The foregoing proposition and corollary show that $\mathrm{Sz} Q$ behaves quite differently from $\operatorname{Sz} D(L)$, where $D(L)$ denotes the division ring of quotients of a universal enveloping algebra of a finite dimensional Lie algebra $L$. In case of $D(L)$ one has $\operatorname{Sz} D(L) \cong Z D(L)\left[\Lambda_{D}(L)\right][17]$, a group algebra over the torsion-free abelian group of weights $\Lambda_{D}(L)$. In particular $\operatorname{Sz} D(L)$ is finite dimensional over $Z D(L)$ only in case $\Lambda_{D}(L)$ is trivial. This is also the only case in which $\operatorname{Sz} D(L)$ is a field.

\section{Centralizers of semi-invariants}

We prove the analogue of [7, Prop. 1.15 and Cor. 1.16] in the case of a group algebra.

Denote $G_{\Lambda}=\bigcap_{\lambda \in \Lambda(G)} \operatorname{ker} \lambda$.

Proposition 3.1. Let $\propto$ be a semi-invariant of $K[G]$ with weight $\lambda$. Denote $H=\operatorname{ker} \lambda$. Then

(1) $C_{K[G]}(\alpha)=K[H]$;

(2) $C_{Q}(\alpha)=Q_{c l}(K[H])$.

Proof. Since $H$ has finite index in $G$ we have $\Delta(H) \subset \Delta(G)$; conversely $\Delta(G) \subset$ $C_{G}(\Delta) \subset$ ker $\lambda=H$ by Lemma 1.3. Thus $\Delta(H)=\Delta(G)$. Also note that by Proposition $1.2 \alpha \in K[\Delta(G)]=K[\Delta(H)] \subset K[H]$.

(1) Clearly $K[H] \subset C_{K[G]}(\alpha)$. To prove the converse inclusion, let $\left\{g_{1}=1, g_{2}, \ldots, g_{n}\right\}$ be a transversal for $H$ in $G$. Then $K[G]=\oplus_{i=1}^{n} K[H] g_{i}$ and each element $u \in K[G]$ can be written in a unique way as $u=\sum_{i=1}^{n} u_{i} g_{i}$ where $u_{i} \in K[H]$ for all $i$. Let $u \in C_{K[G]}(\alpha)$ and write $u=\sum_{i=1}^{n} u_{i} g_{i}$ as before. Using the fact that $\alpha \in(K[G])_{\lambda}$, and that $\alpha$ and all $u_{i}$ belong to $K[H]$, we obtain 


$$
\begin{aligned}
0 & =u \alpha-\alpha u \\
& =\sum u_{i} g_{i} \alpha-\sum \alpha u_{i} g_{i} \\
& =\sum u_{i} \lambda\left(g_{i}\right) \alpha g_{i}-\sum u_{i} \alpha g_{i} \\
& =\sum u_{i} \alpha\left(\lambda\left(g_{i}\right)-1\right) g_{i}
\end{aligned}
$$

and thus $u_{i} \alpha\left(\lambda\left(g_{i}\right)-1\right)=0$ for all $i$. If $i \neq 1$, then $\lambda\left(g_{i}\right) \neq 1$ since $g_{i} \notin \operatorname{ker} \lambda=H$. Thus $u_{i} \alpha=0$ and hence $u_{i}=0$ because $\alpha$ is a regular element. This shows that $u=u_{1} \in K[H]$.

(2) Clearly $Q_{c l}(K[H]) \subset C_{Q}(\alpha)$. Conversely, let $u \in C_{Q}(\alpha)$. By [19, Lemma 13.3.5]. $Q_{c l}(K[G])=\left\{\beta^{-1} \gamma \mid \gamma \in K[G], \beta\right.$ a regular element of $\left.K[H]\right\}$. Thus $\beta u=\gamma$ for some $\gamma \in K[G]$ and some regular element $\beta \in K[H]$. Since $\beta$ and $u$ commute with $\alpha$, the same holds for $\gamma$ and by (1) $\gamma \in K[H]$. Therefore $u \in Q_{c l}(K[H])$.

Proposition 3.2. (1) $C_{K[G]}(\mathrm{Sz} K[G])=K\left[G_{\Lambda}\right]$;

(2) $C_{Q}(\operatorname{Sz} K[G])=Q_{c l}\left(K\left[G_{\Lambda}\right]\right)$

Proof. (1) $C_{K \mid G)}(\mathrm{Sz} K[G])=\bigcap_{z}$ semi-invariant $C_{K[G]}(\alpha)=\bigcap_{\lambda \in \Lambda(G)} K[\operatorname{ker} \lambda]=K\left[G_{\Lambda}\right]$;

(2) This is shown in the same way as (1), using again [19, Lemma 13.3.5].

\section{Structure of $\mathrm{Sz} K[G]$}

We already know by Proposition 1.2 that $\mathrm{SzK}[G]$ is a commutative domain. In this section we show that $\mathrm{SzK}[G]$ has a much richer structure. To simplify notations we will denote $C_{G}(\Delta)$ in this section by $C$.

Lemma 4.1. $Z K[G]=K[\Delta]^{G}=K[\Delta]^{\bar{G}}$ where $\bar{G}=G / C$.

Proposition 4.2. $\mathrm{SzK}[G]=K[\Delta]^{G_{\Lambda}}=K[\Delta]^{\bar{\epsilon}_{\Lambda}}$ where $\bar{G}_{\Lambda}=G_{\Lambda} / C$.

Proof. The proof is a slight change of the proof of [22, Lemma 3]; for the sake of completeness we include the main details. First note that $G / G_{\wedge}$ is a finite abelian group because $G^{\prime} \subset G_{\Lambda}$ and $C_{G}(\Delta) \subset G_{\Lambda}$. If $G_{\Lambda}=G$, then $\lambda\left(G_{\Lambda}\right)=\lambda(G)=1$ for all $\lambda \in \Lambda(G)$, i.e., $\lambda=1$. In particular $\Lambda(G)=1$ and thus $\operatorname{SzK}[G]=Z K[G]=K[\Delta]^{G}=K[\Delta]^{G_{\Lambda}}$ by Lemma 4.1 .

If $G_{\Lambda} \neq G$, we claim that $G / G_{\Lambda}$ is $K$-complete. Let $x \in G \backslash G_{\Lambda}$; then for some $\lambda \in \Lambda(G)$ we have $\lambda(x) \neq 1$. Since $\lambda\left(G_{\Lambda}\right)=1$, the map $\lambda: G \rightarrow K^{*}$ can be lifted to $\bar{\lambda}: G / G_{\Lambda} \rightarrow K^{*}$. So $\bar{\lambda}\left(x G_{\Lambda}\right)=\lambda(x) \neq 1$. Thus $G / G_{\Lambda}$ is $K$-complete.

By definition of $G_{\wedge}$ it is obvious that $\operatorname{SzK}[G] \subset K[\Delta]^{G_{\Lambda}}$. To prove the converse inclusion, let $a \in \Delta$; if $C(a)$ denotes the centralizer of $a$ in $G$, then $C=C_{G}(\Delta) \subset C(a)$. 
Let $Q$ be a (finite) transversal for $C$ in $G_{\Lambda}$. Define $s_{a}=\sum_{q \in Q} a^{q}$; clearly $s_{a}$ is independent of the choice of $Q$; moreover $K[\Delta]^{G_{\Lambda}}$ is spanned by elements of the form $s_{a}$. Let $\lambda \in \Lambda(G)$ and $U$ be any transversal for $C$ in $G$. Define $s_{2}=\sum_{\sigma \in U} \lambda\left(\sigma^{-1}\right) a^{\sigma}$. Then $s_{\lambda}$ is independent of the choice of $U$ and $s_{i} \in K[G]_{i}$. In particular, if $P=\left\{p_{1}=1, \ldots, p_{n}\right\}$ is a transversal for $G_{\wedge}$ in $G$ and $Q=\left\{q_{1}, \ldots, q_{m}\right\}$ is defined as before, then $P Q$ is easily seen to be a transversal for $C$ in $G$. A straightforward calculation as in [22, Lemma 3] shows that

$$
s_{\lambda}=\sum_{i=1}^{n} \lambda\left(p_{i}^{-1}\right) s_{a}^{p_{i}}
$$

As mentioned before, $\lambda$ can be lifted to $\bar{\lambda}: G / G_{\Lambda} \rightarrow K^{*}$. Since $G / G_{\Lambda}$ is $K$-complete, by $\left[19\right.$, Lemma 4.3.3] there exist $\bar{\lambda}_{1}, \ldots, \bar{\lambda}_{n} \in \operatorname{Hom}\left(G / G_{\Lambda}, K^{*}\right)$ such that $\operatorname{det}\left(\lambda_{i}\left(p_{j}^{-1}\right)\right) \neq 0$. By elementary linear algebra each $s_{a}^{p_{i}}$ is a linear combination of $s_{\lambda_{1}}, \ldots, s_{\lambda_{n}}$, thus belongs to $\operatorname{SzK}[G]$. In particular $s_{a}=s_{a}^{p_{1}} \in \operatorname{Sz} K[G]$. This shows that $K[\Delta]^{G_{\Lambda}} \subset \operatorname{Sz} K[G]$.

In the case that the field of coefficients is algebraically closed, M. Smith proved that the result of Proposition 4.2 can be sharpened - and simplified - by replacing $G_{\Lambda}$ by $G^{\prime}$.

Proposition 4.3. (1) $\mathrm{Sz} \bar{K}[G]=\bar{K}[\Delta]^{G^{\prime}}=\bar{K}[\Delta]^{G C / C}$;

(2) $\mathrm{Sz} \bar{K}[G] \cap K[G]=K[\Delta]^{G^{\prime}}=K[\Delta]^{G^{\prime} C / C}$.

Proof. (1) As mentioned, this is proved in [22, Lemma 3].

(2) By (1) $S z \bar{K}[G] \cap K[G]=\bar{K}[\Delta]^{G} \cap K[G]=K[\Delta]^{G^{\prime}}$.

Example 6.4 shows that it is possible that $\mathrm{SzK}[G] \varsubsetneqq(\mathrm{Sz} \bar{K}[G] \cap K[G])$. In some cases however, equality holds.

Lemma 4.4. Let $K$ and $L$ be fields with $K \subset L$. Write $L=\oplus_{i \in I} a_{i} K$ for some index set $I$ and choose $a_{1}=1$. If $\Lambda=\Lambda(G, K)=\Lambda(G, L)$ (i.e., $\lambda(G) \subset K^{*}$ for all $\lambda \in \Lambda(G, L)$ ), then

(1) for each $\lambda \in \Lambda\left(L[G]_{\lambda}=\oplus_{i \in I} a_{i}\left(K[G)_{\lambda}\right.\right.$;

(2) $\mathrm{Sz} L(G) \cap K[G]=\mathrm{Sz} K[G]$.

Proof. Obvious.

Corollary 4.5. If $\Lambda(G, K)=\Lambda(G, \bar{K})$, then $\operatorname{Sz} K[G]=\operatorname{Sz}(\bar{K}[G]) \cap K[G]=K[\Delta]^{G^{\prime}}$.

Corollary 4.6. Given a field $K$ there exists a finite extension $L$ of $K$ such that $\mathrm{Sz} L[G]=L[\Delta]^{G^{\prime}}$ 
Proof. Consider $\Lambda(G, \bar{K})$, which is a finite group by Proposition 1.6. Write $\Lambda(G, \bar{K})=\left\{\lambda_{1}, \ldots, \lambda_{m}\right\}$ for some $m$. Also $G$ is finitely generated, say by $g_{1}, \ldots, g_{n}$ for some $n$. Let $L$ be the field generated by $K$ and all $\lambda_{i}\left(g_{j}\right)$, where $1 \leq i \leq m$ and $1 \leq j \leq n$. Since each $\lambda_{i}\left(g_{j}\right) \in \bar{K}^{*}$, it is clear that $L$ is a finite dimensional extension of $K$. By construction of $L$ we have $\lambda_{i}(G) \subset L^{*}$ for all $i$. Thus $\Lambda(G, \bar{K})=\Lambda(G, L)$. The result follows now from Corollary 4.5 .

Proposition 4.7. $Z K[G], \mathrm{Sz} K[G]$ and $\mathrm{Sz} \bar{K}[G] \cap K[G]$ are finitely generated $K$-algebras and Noetherian Krull domains.

Proof. By $4.1,4.2$ and 4.3 the rings mentioned above are fixed rings of $K[\Delta]$ under a finite group. Noether's theorem implies that these rings are finitely generated $K$ algebras and thus Noetherian. Since

$$
Z K[G]=K[\Delta]^{G}=K[\Delta] \cap L^{G},
$$

where $L$ is the field of fractions of $K[\Delta]$, it is trivial to see that $Z K[G]$ is a Krull domain. For the two other rings, the proof is similar.

In contrast to Lie algebras, an example due to the author which appeared in [17] shows that $Z U(L)$ and $\mathrm{Sz} U(L)$ need not be Noetherian. We have shown now that $Z K[G], S z K[G]$ and $\mathrm{Sz}(\bar{K}[G]) \cap K[G]$ are Krull domains. A natural question is whether these rings are also $U F D$ 's, and if not in general when are they a $U F D$ ? Note that in the case of a universal enveloping algebra $U(L)$ of a finite dimensional Lie-algebra $L$, the semicentre $\operatorname{Sz} U(L)$ is always a $U F D$, as is well-known [12, 9].

Therefore S. Montgomery asked whether the semicentre of a prime group algebra is a $U F D$, as is mentioned in the introduction of [22]. M. Smith answers this question in the negative sense. In the same paper [22], M. Smith states that it may be of interest to determine necessary and sufficient conditions for $S z K[G]$ to be a $U F D$. In particular she asks whether $\operatorname{Sz} K[G]$ is a $U F D$ in case $K[G]$ is a $U F R$ in the sense of Chatters and Jordan [6]. We show that the example of a group algebra $K[G]$ - given by $M$. Smith in [22] - such that $\mathrm{SzK}[G]$ is not a $U F D$, is such that $G$ is polycyclic-by-finite and $K[G]$ is a prime $U F R$ (cf. Example 6.3).

Quite recently, $M$. Lorenz described the class group of a ring of multiplicative invariants [10]. To be a little more precise, recall that $\operatorname{Sz} K[G]=K[\Delta]^{\bar{G}_{A}}$ where $\bar{G}_{\Lambda}=G_{\Lambda} / C$ is a finite group which acts on the finitely generated free abelian group $\Delta$. By identifying $\Delta$ with $\mathbb{Z}^{d}$ for some $d, \bar{G}_{\Lambda}$ becomes a finite subgroup of $G L_{d}(\mathbb{Z}) \cong G L(\Delta)$. In particular $S L(\Delta) \cong S L_{d}(\mathbb{Z})$ are the elements of $G L_{d}(\mathbb{Z})$ having determinant 1 . If $N$ denotes the (normal) subgroup of $\bar{G}_{\Lambda}$ generated by all the reflections in $\bar{G}_{\Lambda}$ and $D$ the (normal) subgroup generated by the reflections that are diagonalisable over $\mathbb{Z}$, then the following result holds.

Proposition 4.8 (M. Lorenz [10]). The class group of $\mathrm{SzK}[G]$ is isomorphic to 


$$
\operatorname{Cl}(\mathrm{SzK}[G]) \cong \operatorname{Hom}\left(\bar{G}_{\Lambda} / N, K^{*}\right) \oplus H^{1}\left(\bar{G}_{\Lambda} / D, \Delta^{D}\right)
$$

By replacing $\bar{G}_{\Lambda}$ by $\bar{G}$ (resp. $G^{\prime} C / C$ ) one obtains a similar result for the class group of $Z K[G]$ and $\mathrm{Sz}(\bar{K}[G]) \cap K[G]$. The formula in Proposition 4.8 already indicates that the property of being a $U F D$ will not only depend on $G$ but also on the field $K$.

The following result is due to $K$. A. Brown and M. Lorenz [5] and is somewhat weaker than Proposition 4.8, but will turn out to be more practical in some concrete cases.

Proposition 4.9 (K. A. Brown, M. Lorenz [5]). (1) $C l(Z K[G])$ is a subgroup of $\operatorname{Hom}\left(\bar{G}, K^{*}\right) \times H^{1}(\bar{G}, \Delta)$;

(2) $C l(\mathrm{SzK}[G])$ is a subgroup of $\operatorname{Hom}\left(\bar{G}_{\Lambda}, K^{*}\right) \times H^{1}\left(\bar{G}_{\Lambda}, \Delta\right)$;

(3) $C l(\operatorname{Sz}(\bar{K}[G]) \cap K[G])$ is a subgroup of $\operatorname{Hom}\left(G^{\prime} C / C, K^{*}\right) \times H^{1}\left(G^{\prime} C / C, \Delta\right)$;

(4) if $K$ is algebraically closed, then $C l(\operatorname{Sz} K[G])$ is a subgroup of $\operatorname{Hom}\left(G^{\prime} C / C, K^{*}\right) \times$ $H^{1}\left(G^{\prime} C / C, \Delta\right)$.

Corollary 4.10. (1) If $\operatorname{Hom}\left(\bar{G}, K^{*}\right)=\{1\}$ and $H^{1}(\bar{G}, \Delta)=\{1\}$, then $\operatorname{Sz} K[G]=Z K[G]$ and is a UFD;

(2) If $(G: C)$ is odd and $H^{\prime}(\bar{G}, \Delta)=\{1\}$, then $\operatorname{Sz} \mathbb{R}[G]=Z \mathbb{R}[G]$ and is a UFD.

Proof. (1) This is obvious by Corollary 1.7 and Proposition 4.9(1).

(2) An elementary calculation shows that $\operatorname{Hom}\left(\bar{G}, \mathbb{R}^{*}\right)=\{1\}$ because $|\bar{G}|$ is odd.

\section{Normal elements versus semi-invariants}

If $L$ is a finite dimensional Lie-algebra, then $u \in U(L)$ is a normal element if and only if $u$ is a semi-invariant (see [7, Proposition 1.8] or [21, Corollary 13.8]). In case of a group algebra $K[G]$ this is no longer true, because any unit in $K[G]$ is trivially a normal element but a unit $u$ is only a semi-invariant if $u=k g$ where $k \in K^{*}$ and $g \in Z(G)$. Therefore the best we can hope is that every normal element is the product of a unit and a semi-invariant. In general this will not be the case. In Theorem 5.3 we will prove necessary and sufficient conditions such that every normal element which belongs to $K[\Delta]$ is the product of an element of $\Delta$ and a semi-invariant. In case $K[G]$ is a $U F R$ in the sense of Chatters and Jordan, the restriction to normal elements belonging to $K[\Delta]$ won't be a real restriction.

Lemma 5.1. (1) If $\alpha$ is a normal element of $K[G]$, then for each $g \in G$ there exists a unit $v_{g}$ of $K[G]$ such that $\alpha^{g}=g \alpha g^{-1}=\alpha v_{g}$.

(2) If $\alpha$ is a normal element belonging to $K[\Delta]$, then $v_{g}=k_{g} u_{g}$ where $k_{g} \in K^{*}$ and $u_{g} \in \Delta$. 
Proof. (1) Let $g \in G$; since $\alpha$ is normal

$$
\left(g \alpha g^{-1}\right) K[G]=g \alpha K[G]=g K[G] \alpha=K[G] \alpha=\alpha K[G]
$$

and the fact that $\alpha$ and $g \alpha g^{-1}$ are regular implies that $g \alpha g^{-1}=\alpha v_{g}$ for some unit $v_{g}$ of $K[G]$.

(2) If $\alpha$ is normal and $\alpha \in K[\Delta]$, then $v_{g} \in K[\Delta]$ because $\alpha^{g} \in K[\Delta]$. The fact that $v_{g}$ is a unit in $K[\Delta]$ and $\Delta$ is torsion-free abelian implies that $v_{g}=k_{g} u_{g}$ for some $k_{g} \in K^{*}$ and $u_{g} \in \Delta$.

Up to a slight difference in notation, the following lemma is proved in $[15$, Lemma 2] and [16, Lemma 1(i)].

Lemma 5.2. Let $\sigma \in$ Aut $G$ centralise a subgroup of finite index. Let $W=C_{G}(\sigma)=\{g \in G \mid \sigma(g)=g\}$ and $T$ be a left transversal of $W$ in $G$. Denote

$$
\alpha=\sum_{i \in T} t^{\sigma} t^{-1}
$$

Then $\alpha$ is a normal element of $K[G]$ belonging to $K[\Delta]$ such that $g^{\sigma} \alpha g^{-1}=\alpha$ for all $g \in G$.

Theorem 5.3. The following conditions are equivalent:

(1) every normal element of $K[G]$ belonging to $K[\Delta]$ can be written as us where $u \in \Delta$ and $s$ is a semi-invariant;

(2) if $\sigma \in$ Aut $G$ centralise a subgroup $H$ of finite index, then $\sigma$ is an inner automorphism of $G$;

(3) $H^{1}\left(G / C_{G}(\Delta), \Delta\right)=\{1\}$

In the case that these conditions are satisfied, the decomposition of a normal element into a product of an element of $\Delta$ and a semi-invariant is unique up to a central element of $G$.

Proof. (1) $\Rightarrow(2)$ : Let $\sigma \in$ Aut $G$ be such that $\sigma$ centralises a subgroup of finite index. If $W=C_{G}(\sigma)$, then $(G: W)$ is finite. Using the result and the notations as in Lemma 5.2, $\alpha=\sum_{t \in T} t^{\sigma} t^{-1}$ is a normal element of $K[G]$ belonging to $K[\Delta]$. By (1) $\alpha$ can be written as $\alpha=u s$, where $u \in \Delta$ and $s \in(K[G])$, for some weight $\lambda$. For all $g \in G$

$$
\alpha^{g}=u^{g} s^{g}=\lambda(g) u^{g} s=\lambda(g) u^{g} u^{-1} \alpha
$$

By Lemma 5.2

$$
\alpha^{g}=g\left(g^{-1}\right)^{\sigma} g^{\sigma} \alpha g^{-1}=g\left(g^{-1}\right)^{\sigma} \alpha
$$


A combination of $(*)$ and $(* *)$, using the fact that $\alpha$ is regular, yields

$$
g\left(g^{-1}\right)^{\sigma}=\lambda(g) u^{g} u^{-1}
$$

For a start this implies that $\lambda(G)=\{1\}$, i.e., $s$ is a central element. Secondly $g\left(g^{-1}\right)^{\sigma}=u^{g} u^{-1}$ implies $\left(g^{-1}\right)^{\sigma}=u g^{-1} u^{-1}$, i.e., $\sigma$ is an inner automorphism.

(2) $\Leftrightarrow(3)$ : This holds even more generally, as shown by K. A. Brown in [4, p. 85].

(3) $\Rightarrow(1)$ : Let $\alpha$ be a normal element of $K[G]$ belonging to $K[\Delta]$. By Lemma $5.1(2)$, for all $g \in G$ we have $g \alpha g^{-1}=k_{g} u_{g} \alpha$ for some $k_{g} \in K^{*}$ and $u_{g} \in \Delta$. Note that if $g \in C$ then $g \alpha g^{-1}=\alpha$, so $k_{g}=1$ and $u_{g}=1$. Hence the following map is well-defined:

$$
G / C \rightarrow K[G]: \bar{g} \mapsto \alpha^{g}=g \alpha g^{-1}
$$

For all $g, h \in G,(g h) \alpha(g h)^{-1}=k_{g h} u_{g h} \alpha$ and $(g h) \alpha(g h)^{-1}=g\left(h \alpha h^{-1}\right) g^{-1}=g\left(k_{h} u_{h} \alpha\right) g^{-1}=$ $k_{g} k_{h} u_{g} u_{h}^{g} \alpha$, which implies that $k_{g h}=k_{g} k_{h}$ and $u_{g h}=u_{g} u_{h}^{g}$. Therefore

$$
f_{\alpha}: G / C \rightarrow \Delta: \bar{g} \mapsto u_{g}
$$

is a 1-cocycle. By (3) $f_{x}$ is a 1-coboundary, so there exists $v \in \Delta$ such that $u_{g}=f_{\alpha}(\bar{g})=v^{-1} v^{\bar{g}}$ for all $\bar{g} \in G / C$. Let $s=v^{-1} \alpha$. Using the fact that $\Delta$ is abelian a straightforward calculation shows that $s$ is a semi-invariant with weight $k$. Since $\alpha=v s$, this shows (1).

Finally suppose $\alpha=u s=u^{\prime} s^{\prime}$, where $u, u^{\prime} \in \Delta$ and $s$ (resp. $s^{\prime}$ ) are semi-invariants with weight $\lambda$ (resp. $\lambda^{\prime}$ ). Then $s=u^{\prime \prime} s^{\prime}$ where $u^{\prime \prime}=u^{-1} u^{\prime} \in \Delta$. Using the fact that $s$ and $s^{\prime}$ are semi-invariants we obtain

$$
\lambda(g) s=s^{g}=u^{\prime \prime g} s^{g}=\lambda^{\prime}(g) u^{\prime \prime g} s^{\prime}=\lambda^{\prime}(g) u^{\prime \prime g} u^{\prime \prime-1} s,
$$

which implies that $\lambda=\lambda^{\prime}$ and $u^{\prime \prime g}=u^{\prime \prime}$ for all $g \in G$, i.e., $u^{\prime \prime} \in Z(G)$.

Proposition 5.4. Let $K[G]$ be a UFR such that $H^{1}(G / C, \Delta)=\{1\}$.

(1) Every height one prime ideal $P$ of $K[G]$ is generated by a semi-invariant.

(2) A normal element of $K[G]$ is the product of a unit of $K[G]$ and a semi-invariant.

(3) Every semi-invariant can uniquely (up to an element of $K^{*}$ and of $Z(G)$ ) be written as a product of irreducible semi-invariants.

Proof. Note first that a Noetherian $U F R$ is a maximal order [6, Theorem 2.4]. In this case a divisorial prime ideal is the same as a height one prime ideal. Moreover the group of divisorial ideals is a free abelian group generated by the height one prime ideals.

(1) This is clear from the fact that $K[G]$ is a $U F R$, that $P$ is generated by a normal element belonging to $K[\Delta]$ [3, Theorem $B]$ and Theorem 5.3. 
(2) Let $p$ be a normal element of $K[G]$. If $p$ is not a unit of $K[G]$ then $K[G] p$ is a divisorial ideal of $K[G]$. Using the fact that each height one prime ideal $P$ is generated by a semi-invariant $s$, we have

$$
p=u s_{1} \ldots s_{n}
$$

for some $n, u$ a unit of $K[G]$ and each $s_{i}$ a semi-invariant.

(3) This is proved in the same way as (2), using the fact that in the expression $s=u s_{1} \ldots s_{n}$ the unit $u$ of $K[G]$ belongs to $K[\Delta]$ and is a semi-invariant, thus $u \in K^{*} \cdot Z(G)$. The uniqueness of this decomposition follows from the fact that $K[G]$ is a $U F R$.

\section{Examples}

In this section we give a number of examples which either illustrate some of the results in the foregoing section or either illustrate some differences with the semicentre of a universal enveloping algebra of a Lie-algebra, especially concerning the question of when the semicentre is a UFD.

6.1 Example 1. Let $G$ be the group generated by $x$ and $y$ such that $y x y^{-1}=x^{-1}$. Clearly $G$ is torsion-free poly-infinite cyclic, so $K[G]$ is a Noetherian domain which is a maximal order by $[3$, Theorem $\mathrm{F}]$ and even a $U F R$, because $K[G]$ is clearly a $P I$-ring, by $\left[3\right.$, Theorems $C$ and D]. As can easily be checked $\Delta=\Delta(G)=C_{G}(\Delta)=C=\left\langle x, y^{2}\right\rangle$ and $G^{\prime}=\left\langle x^{2}\right\rangle$. For any field $K$ we have $\operatorname{Hom}\left(G / C, K^{*}\right)$ is cyclic of order two; thus by Corollary $4.5 \mathrm{Sz} K[G]=K[\Delta]^{G^{\prime}}=K[\Delta]$, because $G^{\prime} \subset C$.

Using the fact and notation that $G / C \cong\langle\bar{y}\rangle$ where $\bar{y}^{2}=1$, we obtain by direct calculation that a map $\varphi$ from $G / C$ to $\Delta$ is a 1 -cocycle if $\varphi(\bar{y})=x^{i}(i \in \mathbb{Z})$ and $\varphi$ is a 1-coboundary if $\varphi(\bar{y})=x^{2 i}(i \in \mathbb{Z})$. Thus $H^{1}(G / C, \Delta) \cong C_{2}$. By Theorem 5.3, not every normal element of $K[G]$ which belongs to $K[\Delta]$ can be written as the product of an element of $\Delta$ and a semi-invariant. A concrete example is the following: let $p=1+x$; as is directly checked $p$ is a normal element belonging to $K[\Delta]$. Using the fact that $y p y^{-1}=x^{-1} p$, the map $\varphi_{p}: G / C \rightarrow \Delta$ is such that $\varphi_{p}(\bar{y})=x^{-1}$ is a 1-cocycle but not a 1-coboundary. A straightforward calculation shows that a normal element $p$ which can be written as $u s$ where $u \in \Delta$ and $s$ a semi-invariant induces a 1 coboundary $\varphi_{p}$.

A useful property in enveloping algebras of finite dimensional Lie-algebras is the fact that $u$ and $v$ are semi-invariants if $u v$ is a semi-invariant [13]. This need not hold anymore for group algebras $K[G]$. Consider this group $G$. Let $u=(1+x) y$; then $u^{2}=\left(2+x+x^{-1}\right) y^{2} \in Z K[G]$. Let $v=2+x$ and $w=2+x^{-1}$, then $v w=$ $5+2\left(x+x^{-1}\right) \in Z K[G]$. In particular $u^{2}$ and $v w$ are semi-invariants but neither $u, v$ or $w$ is a semi-invariant, in fact $v$ and $w$ are not even normal elements. 
6.2 Example 2. This example can be found in [11, pp. 383-384]. Let $A$ be the free abelian group with basis $a, b, c$ and $d$, and let $\langle z\rangle$ be an infinite cyclic group acting on $A$ via an automorphism $\varphi_{z}$, where $\varphi_{z}(a)=a, \varphi_{z}(b)=b^{-1} a, \varphi_{z}(c)=c b$ and $\varphi_{z}(d)=d^{-1} c$. Let $G$ be the semidirect product of $A$ and $\langle z\rangle$. As mentioned in [11], $G$ is torsion-free nilpotent-by-finite and by [11, Proposition 5.4] every nonzero ideal of $K[G]$ intersects $Z K[G]$ nontrivially. By $[3$, Theorems $\mathrm{C}$ and $\mathrm{D}], K[G]$ is a $U F R$. One has $\Delta=\Delta(G)=\langle a, b\rangle$ and $C=C_{G}(\Delta)=\left\langle A, z^{2}\right\rangle$; thus $G / C \cong\langle\bar{z}\rangle$ and $\bar{z}^{2}=1$, which implies that $\Lambda(G, K) \cong \operatorname{Hom}\left(G / C, K^{*}\right) \cong C_{2}$ for all fields $K$. On the other hand, $\Delta \subset G^{\prime}=\left\langle\Delta, c^{-1} d^{2}\right\rangle \subset C$. Corollary 4.5 implies that $\operatorname{Sz} K[G]=K[\Delta]^{\sigma^{\prime}}=K[\Delta]$ for any field $K$. A straightforward calculation shows that every 1 -cocycle from $G / C$ to $\Delta$ is a 1-coboundary. Thus $H^{1}(G / C, \Delta)=\{1\}$ and the results of Theorem 5.3 and Proposition 5.4 apply.

6.3 Example 3. This example appears in [22, Example 1], in which the author proves that the semicentre is not a $U F D$ in case $K$ is algebraically closed. We will show precisely for which fields the semicentre is a $U F D$; in the other case we will compute the class group of the semicentre. The next two examples are variants of this construction.

Let $A$ be free abelian on $a_{1}, b_{1}, c_{1}, a_{2}, b_{2}, c_{2}$ and let $H$ be generated by $\sigma$ and $\tau$ such that $\sigma \tau \sigma^{-1}=\tau^{-1}$ ( $H$ is thus the group used in Example 1). Let $\varphi: H \rightarrow$ Aut $A$ be a homomorphism defined by $\varphi(\sigma)$ (denoted in brief by $\varphi_{\sigma}$ ) for which $\varphi_{\sigma}\left(a_{i}\right)=b_{i}$, $\varphi_{\sigma}\left(b_{i}\right)=a_{i}, \varphi_{\sigma}\left(c_{i}\right)=c_{i}(i \in\{1,2\})$ and $\varphi_{\tau}$ is defined by cyclic permutation of $a_{1}, b_{1}, c_{1}$ and $a_{2}, b_{2}, c_{2}$. Let $G$ be the semidirect product of $A$ and $H$. Clearly $G$ is torsion-free poly-infinite cyclic. Then $\Delta=\Delta(G)=\left\langle A, \sigma^{2}, \tau^{3}\right\rangle$. Thus $(G: \Delta)$ is finite, $G$ is abelian-byfinite and $K[G]$ is a $P I$-ring. Using [3, Theorems $C$ and $D], K[G]$ is a $U F R$. Moreover $C=C_{G}(\Delta)=\Delta$ and $G / C$ is isomorphic to the symmetric group of degree 3 . Then $\Lambda(G, K) \cong \operatorname{Hom}\left(G / C, K^{*}\right) \cong C_{2}$ for any field $K$. Then $\bar{G}_{\Lambda} \cong\langle\bar{\tau}\rangle$ is cyclic of order three and we have $\bar{G}_{\Lambda} \subset S L(\Delta)$, as is readily checked (the notation $S L(\Delta)$ is mentioned just before Proposition 4.8). In particular $\bar{G}_{\Lambda}$ contains no reflections, since a reflection has determinant -1 (see e.g., [10]). To show that $H^{1}\left(\bar{G}_{\Lambda}, \Delta\right)=\{1\}$, let $f$ be a 1 -cocycle defined by $f(\bar{\tau})=p \sigma^{2 i} \tau^{3 j}$, where $p \in A$ and $i, j, \in \mathbb{Z}$. Using the fact that $\bar{\tau}^{3}=1$, direct calculation shows that $i=j=0$ and $f(\bar{\tau})=p=\left(a_{1} c_{1}^{-1}\right)^{\alpha_{1}}\left(b_{1} c_{1}^{-1}\right)^{\beta_{1}}\left(a_{2} c_{2}^{-1}\right)^{\alpha_{2}}\left(b_{2} c_{2}^{-1}\right)^{\beta_{2}}$ for some $\alpha_{1}, \alpha_{2}, \beta_{1}, \beta_{2} \in \mathbb{Z}$. Let $u=b_{1}^{-\beta_{1}} c_{1}^{\alpha_{1}} b_{2}^{-\beta_{2}} c_{2}^{\alpha_{2}}$; then $f(\bar{\tau})=u^{-1} u^{\bar{\tau}}$, i.e., $f$ is a 1-coboundary. Proposition 4.8 implies that $C l(\operatorname{SzK}[G]) \cong \operatorname{Hom}\left(\bar{G}_{\Lambda}, K^{*}\right)$. Obviously $\operatorname{SzK}[G]$ is a $U F D$ if and only if $K$ does not contain a primitive third root of unity. In the other case $C l(\mathrm{Sz} K[G]) \cong C_{3}$.

6.4 Example 4. This example is a slight variation of the foregoing example. Let again $A$ be the free abelian group on $a_{1}, b_{1}, c_{1}, a_{2}, b_{2}, c_{2}$ and $H=\langle\tau\rangle$ be infinite cyclic. Let $\varphi: H \rightarrow$ Aut $A$ be defined by $\varphi(\tau)$ which permutes $\left\{a_{1}, b_{1}, c_{1}\right\}$ and $\left\{a_{2}, b_{2}, c_{2}\right\}$ cyclically. Let $G$ be the semidirect product of $A$ and $H$. Then $\Delta=\Delta(G)=\left\langle A, \tau^{3}\right\rangle=$ $C_{G}(\Delta)=C$ and $\left.G^{\prime}=\left\langle a_{i}^{-1} b_{i}, b_{i}^{-1} c_{i}\right| i \in\{1,2\}\right)$. Note that $G^{\prime} \subset C$. Since $G / C \cong C_{3}$, we have $\Lambda(G, K) \cong \operatorname{Hom}\left(G / C, K^{*}\right)=\{1\}$ if $K$ does not contain a primitive third root of unity; in the other case $\operatorname{Hom}\left(G / C, K^{*}\right) \cong C_{3}$. A computation similar to the one in Example 6.3 shows that $H^{1}(G / C, \Delta)=\{1\}$. 
This leads to the following result. If $K$ does not contain a primitive third root of unity, then Corollary 4.10 implies that $\operatorname{SzK}[G]=Z K[G]$ is a $U F D$. If $K$ does contain a primitive third root of unity $\Lambda(G, K)=\Lambda(G, \bar{K})$, so $\mathrm{Sz} K[G]=K[\Delta]^{\sigma^{\prime}}=K[\Delta]$ by Corollary 4.5 and the fact that $G^{\prime} \subset \Delta$. Since $\bar{G}=G / C \cong C_{3}$ and, as in Example 6.3, $\bar{G} \subset S L(\Delta)$, so that $\bar{G}$ contains no reflections. Proposition 4.8 used for $Z K[G]$ shows that $C l(Z K[G]) \cong \operatorname{Hom}\left(\bar{G}, K^{*}\right) \oplus H^{1}(\bar{G}, \Delta) \cong C_{3}$. In this example $\operatorname{SzK}[G]$ is a $U F D$ for all fields while $Z K[G]$ is not. Finally, $\operatorname{Sz} K[G] \varsubsetneqq \mathrm{Sz}(\bar{K}[G]) \cap K[G]$ if $K$ does not contain a primitive third root of unity.

6.5 Example 5. Let $A$ be the free abelian group on $x_{1}, y_{1}, x_{2}, y_{2}$ and let $H=\langle\sigma\rangle$ be infinite cyclic. Let $\varphi: H \rightarrow$ Aut $A$ be defined by $\varphi(\sigma)\left(x_{i}\right)=y_{i}^{-1}$ and $\varphi(\sigma)\left(y_{i}\right)=x_{i}$ where $i \in\{1,2\}$. Let $G$ be the semidirect product of $A$ and $H$. Then $\Delta=\Delta(G)=\left\langle A, \sigma^{4}\right\rangle=$ $C_{G}(\Delta)=C$ and $G^{\prime}=\left\langle x_{1} y_{1}, x_{1} y_{1}^{-1}, x_{2} y_{2}, x_{2} y_{2}^{-1}\right\rangle(\subset \Delta)$. Since $G / C$ is cyclic of order 4 , we have $\operatorname{Hom}\left(G / C, K^{*}\right) \cong C_{2}$ if $K$ does not contain a primitive 4 th root of unity and $\operatorname{Hom}\left(G / C, K^{*}\right) \cong C_{4}$ if $K$ does contain a primitive 4 th root of unity. In the last case, $\Lambda(G, K)=\Lambda(G, \bar{K})$ and thus by Corollary $4.5 \mathrm{Sz} K[G]=K[\Delta]^{G^{\prime}}=K[\Delta]$, which clearly is a $U F D$. If $K$ does not contain a primitive 4th root of unity, we claim that $\operatorname{SzK}[G]$ is not a $U F D$. Since $\Lambda(G, K) \cong C_{2}$, we have $\bar{G}_{\Lambda}=G_{\Lambda} / C \cong\left\langle\overline{\sigma^{2}}\right\rangle \cong C_{2}$. Now $\bar{G}_{\Lambda} \subset S L(\Delta)$ :

$$
\begin{aligned}
& \bar{G}_{\wedge} \rightarrow S L(\Delta) \\
& \overline{\sigma^{2}} \mapsto\left(\begin{array}{ccccc}
-1 & & & & 0 \\
& -1 & & & \\
& & -1 & & \\
& & & -1 & \\
0 & & & & \\
0
\end{array}\right)
\end{aligned}
$$

and therefore $\bar{G}_{\Lambda}$ contains no reflections. By Proposition $4.8 \mathrm{Cl}(\mathrm{Sz} K[G]) \cong$ $\operatorname{Hom}\left(\bar{G}_{\Lambda}, K^{*}\right) \oplus H^{1}\left(\bar{G}_{\Lambda}, \Delta\right)$ and $\operatorname{Hom}\left(\bar{G}_{\Lambda}, K^{*}\right)$ is always nontrivial, thus so is $C l(\operatorname{SzK}[G])$ and $\mathrm{SzK}[G]$ is not a $U F D$. By direct computation we have

$$
\mathrm{Sz} K[G]=K\left[\sigma^{4}, \sigma^{-4}\right]\left\langle x_{i}^{k} y_{j}^{\ell}+x_{i}^{-k} y_{j}^{-\ell} \mid i, j \in\{1,2\}, k, \ell \in \mathbb{Z}\right\rangle
$$

Comparing Examples 6.3 and 6.5 leads to the following observation. Let $G$ be the group as in Example 6.3; then $\operatorname{Sz} \mathbb{R}[G]$ is a $U F D$ but $S z \mathbb{C}[G]$ is not a $U F D$. In Example 6.5 the converse happens: $\operatorname{Sz} \mathbb{R}[G]$ is not a $U F D$ while $\operatorname{Sz} \mathbb{C}[G]$ is a $U F D$.

6.6 Example 6. If $L$ is a finite dimensional Lie algebra, then $\operatorname{Sz} U(L)$ is never trivial, i.e., equal to $K$, because every non-zero ideal of $U(L)$ contains a semi-invariant. This example shows that for a group algebra $K[G]$ the semicentre can be trivial. This example appears e.g., in [1, p. 195]. Let $A$ be a free abelian group with basis $y$ and $z$ and $H=\langle x\rangle$ be infinite cyclic. Let $H$ act on $A$ by 


$$
H \rightarrow \text { Aut } A: x \mapsto \varphi_{x}
$$

where

$$
\varphi_{x}(y)=y^{e} z^{g} \text { and } \varphi_{x}(z)=y^{f} z^{h}
$$

such that the matrix $\left(\begin{array}{ll}e & f \\ g & h\end{array}\right)$ has no integer eigenvalues. Let $G$ be the semidirect product of $A$ by $H$. A straightforward calculation shows that $\Delta(G)=\{1\}$, thus $\mathrm{Sz} K[G]=Z K[G]=K$. As mentioned in $[1], K[G]$ is not a $U F R$ in the sense of Chatters and Jordan.

\section{REFERENCES}

1. G. Q. Abbasi, S. Kobayashi, H. Marubayashi, and A. Ueda, Noncommutative unique factorization rings, Comm. Algebra 19 (1991), 167-198.

2. D. F. Anderson, Graded Krull domains, Comm. Algebra 7 (1979), 79-106.

3. K. A. Brown, Height one primes of polycyclic group rings, J. London Math. Soc. 32 (1985), 426-438.

4. K. A. Brown, Class groups and automorphism groups of group rings, Glasgow Math. J. 28 (1986), 79-86.

5. K. A. Brown and M. LORENZ, Grothendieck groups of invariant rings and of group rings, J. Algebra 166 (1994), 423-454.

6. A. W. Chatters and D. A. Jordan, Non-commutative unique factorization rings, $J$. London Math. Soc 33 (1986), 22-32.

7. L. Delvaux, E. Nauwelaerts and A. I. Ooms, On the semi-centre of a universal enveloping algebra, J. Algebra 94 (1985), 324-346.

8. D. F. FARKas, Multiplicative invariants, Enseign. Math. 30 (1984), 141-157.

9. L. Le BRUYN and A. I. Ooms, The semicenter of an enveloping algebra is factorial, Proc. Amer. Math. Soc. 93 (1985), 397-400.

10. M. LoRenz, Class groups of multiplicative invariants, J. Algebra 177 (1995), 242-254.

11. M. LoRenz and D. S. Passman, Centers and prime ideals in group algebras of polycyclic-by-finite groups, J. Algebra 57 (1979), 355-386.

12. M. P. Malliavin, Ultraproduit d'algèbres de Lie (LNM 924, 1982), 157-166.

13. C. Moeglin, Factorialité dans les algèbres enveloppantes, C. R. Acad. Sci. Paris Ser. A 282 (1976), 1269-1272.

14. C. Moeglin, Idéaux bilatères dans les algèbres enveloppantes, Bull. Soc. Math. France 108 (1980), 143-186.

15. S. MONTGOMERY and D. S. Passman, $X$-inner automorphisms of group rings, Houston J. Math. 7 (1981), 395-402.

16. S. Montgomery and D. S. Passman, $X$-inner automorphisms of group rings II, Houston J. Math. 8 (1982), 537-544. 
17. E. Nauwelaerts and A. I. Ooms, Weights of semi-invariants of the quotient division ring of an enveloping algebra, Proc. Amer. Math. Soc. 104 (1988), 13-19.

18. A. I. Ooms and P. WAUTers, Primitive extensions of an enveloping algebra, J. Algebra 161 (1993), 392-405.

19. D. S. PAssman, The algebraic structure of group rings (Wiley-Interscience, New York, 1977).

20. D. S. Passman, Computing the symmetric ring of quotients, J. Algebra 105 (1987), 207-235.

21. D. S. Passman, Infinite crossed products (Academic Press Inc., San Diego, 1989).

22. M. Sмiтн, Semi-invariant rings, Comm. Algebra 13 (1985), 1283-1298.

\section{Department of Mathematics \\ Limburgs UNIVERSITAIR CENTRUM \\ DIEPENBEEK \\ BELGIUM \\ E-mail address: pwauters@luc.ac.be}

